\title{
Potencial erosivo da chuva de Cuiabá, MT: Distribuição e correlação com a precipitação pluviométrica ${ }^{1}$
}

\author{
Cristiana 0. S. Almeida'2, Ricardo S. S. Amorim ${ }^{3}$, Eduardo G. Couto ${ }^{3}$, \\ Flávio L. F. Eltz ${ }^{4} \&$ Laurienne E. C. Borges ${ }^{5}$
}

\begin{abstract}
RESUMO
0 objetivo do presente trabalho foi: (a) determinar o fator de erosividade (R) para o município de Cuiabá, seu período de retorno e sua probabilidade de ocorrência; (b) estudar a distribuição anual, sazonal e mensal do índice de erosividade $\left(\mathrm{EI}_{30}\right)$; (c) definir o período de retorno para os valores dos índices anuais de erosividade e para os valores máximos mensais mais erosivos; e (d) correlacionar o $\mathrm{El}_{30}$ médio mensal com o coeficiente de chuva e precipitação mensal. Foram estudadas, de uma série, 889 chuvas individuais consideradas erosivas, nos períodos de 1972 a 1975, 1977 a 1985 e 1995 a 1999; os pluviogramas dessas chuvas foram cotados, digitalizados e, posteriormente, analisados para obtenção do índice de erosividade $\mathrm{El}_{30}$. Para o município de Cuiabá, $\mathrm{MT}$, o fator $\mathrm{R}$ foi de $8.810 \mathrm{MJ} \mathrm{mm} \mathrm{ha-1} \mathrm{h}^{-1}$ ano-1, classificado alto, com probabilidade de ocorrência de $42,92 \%$, sendo igualado ou superado pelo menos uma vez a cada 2,33 anos. A erosividade das chuvas no período de outubro a abril corresponde a $87 \%$ do total da erosividade anual, indicando ser o período de maior probabilidade de erosão. 0 modelo potencial apresentou melhor ajuste entre $0 \mathrm{El}_{30}$ mensal e 0 coeficiente de chuva.
\end{abstract}

Palavras-chave: erosão hídrica, dados pluviográficos, coeficiente da chuva

\section{Erosive potential of rainfall in Cuiabá, MT: Distribution and correlation with rainfall}

\begin{abstract}
The objectives of this study were: (a) to determine the erosivity factor (R) for the city of Cuiabá, as well as its return period and probability of occurrence, (b) to study the annual distribution, seasonal and monthly index erosivity $\left(\mathrm{El}_{3}\right)$, (c) to define the period of return for the indices of annual erosivity and maximum most erosive monthly values, and (d) to correlate the $\mathrm{El}_{30}$ monthly average with rainfall coefficient and monthly precipitation. A set of 889 erosive individual rains was studied in the period 1972 to 1975 , 1977 to 1985 and 1995 to 1999 , which were digitized and subsequently analyzed to obtain the $\mathrm{El}_{30}$. The $\mathrm{R}$ factor for the Cuiabá city was $8.810 \mathrm{MJ} \mathrm{mm} \mathrm{ha-} \mathrm{h}^{-1}$ year ${ }^{-1}$, classified as high, with probability of $42.92 \%$, being matched or exceeded at least once every 2.33 years. The period from O ctober to A pril concentrated $87 \%$ of total annual erosivity, indicating that it is the period of greatest erosion probability. The potential model showed a better fit between the monthly $\mathrm{El}_{30}$ with the rainfall coefficient.
\end{abstract}

Key words: erosion, rainfall data and rainfall coefficient

\footnotetext{
${ }^{1}$ Parte da Dissertação do primeiro autor, apresentada ao Programa de Pós-Graduação em Agronomia da FAV/U nB, Brasília, DF 2 Engenheira Agrônoma, IN CRA, SIG quadra 04 lote 417/550 CEP 70710-400, Brasília, DF. Fone: (61) 3342-2993. E-mail: kika.df@gmail.com ${ }^{3}$ DSER/U FMT. Av. Fernando Correa da Costa s/n, Campus U FMT. CEP 78060-900 Cuiabá, MT. Fone/Fax: (65) 3615 8616/8668. E-mail: rsamorim@ufmt.br; couto@cpd.ufmt.br

${ }^{4}$ DS/U FSM. Bolsista do CN Pq. Avenida Roraima, n. 1000, Cidade U niversitária, Bairro Camobi, CEP 97105-900 Santa Maria, RS. E-mail: flavioeltz@gmail.com

${ }^{5}$ Graduanda em Engenharia Florestal da UFMT. E-mail: evelyncb21@gmail.com
} 


\section{INTRODUÇÃO}

Uma das principais razões para a diminuição da capacidade produtiva do solo é a erosão, visto que reduz os rendimentos das culturas e lhes causa danos irreversíveis. Um estudo desenvolvido pelo ISRIC/UNEP, do qual participou a Embrapa Solos, indicou que $15 \%$ das terras já foram severamente degradados e $70 \%$ deste total correspondem às perdas da camada superficial do solo (Hernani et al., 2002).

Wischmeier \& Smith (1978) propuseram o modelo USLE, que tem por objetivo prever a erosão que poderá ocorrer em determinada área cultivada, servindo como método para auxiliar na tomada de decisão sobre o uso e o manejo do solo e, consequentemente, relacionar as práticas conservacionistas de maior eficiência. O modelo relaciona, de forma diretamente proporcional, seis fatores que influenciam a erosão hídrica acelerada do solo, que são: erosividade da chuva (R), erodibilidade do solo (K), comprimento e grau do declive (LS), cobertura e manejo do solo (C) e práticas conservacionistas $\operatorname{adotadas}(\mathrm{P})$.

Dentre esses fatores se destaca o fator erosividade da chuva que, segundo Wischmeier \& Smith (1978), é um índice numérico que expressa a capacidade da chuva esperada em dada localidade, de causar erosão em uma área sem proteção. $\mathrm{O}$ parâmetro de erosividade $\mathrm{EI}_{30}$, desenvolvido nos EUA, tem sido calculado e testado em diversos países, sendo sua representação questionada em países tropicais e subtropicais (Moreti et al., 2003a, b; Carvalho et al., 2010).

No Brasil o parâmetro $\mathrm{EI}_{30}$ tem sido amplamente estudado e utilizado. Carvalho et al. (1989), trabalhando em Mococa, SP, encontraram o valor de $7.747 \mathrm{MJ} \mathrm{mm} \mathrm{ha}^{-1} \mathrm{~h}^{-1}$ ano $\mathrm{o}^{-1}$ referente a dezenove anos de dados de precipitação pluvial. Morais et al. (1991) determinaram os índices $\mathrm{EI}_{30}$ médios anuais referentes às estações de Barranquinho e Flechas, do município de Cáceres, sudoeste do Mato Grosso que foram, respectivamente, de 8.493 e $7.830 \mathrm{MJ} \mathrm{mm} \mathrm{ha}^{-1} \mathrm{~h}^{-1}$ ano $^{-1}$. Gonçalves et al. (2006) obtiveram valores mínimos e máximos para oEstado do Rio de Janeiro, obtendo 4.118 MJ mm ha- $\mathrm{h}^{-1}$ ano $^{-1}$ no município de Magé, utilizando uma série de dados de 5 anos e $15.806 \mathrm{MJ} \mathrm{mm}$ $\mathrm{ha}^{-1} \mathrm{~h}^{-1}$ an $^{-1}$ no município de Rio das Flores, com base em dados de 16 anos.

Em conjunto com novos trabalhos em desenvolvimento por pesquisadores nacionais, esses resultados representam um valioso banco de dados para locação das linhas iso-erosivas do Brasil. No Estado de Mato Grosso apenas um trabalho sobre erosividade da chuva foi realizado por Morais et al. (1991), que não trabalharam com pluviogramas e, sim, com dados pluviométricos.

Neste contexto, o objetivo deste trabalho foi: (a) determinar o fator de erosividade para o município de Cuiabá, seu período de retorno e probabilidade de ocorrência; (b) estudar a distribuição anual, sazonal e mensal do índice de erosividade; (c) definir o período de retorno, tanto para os valores dos índices anuais de erosividade como para os valores máximos mensais mais erosivos; e (d) correlacionar o índice de erosividade $\mathrm{EI}_{30}$ médio mensal com o coeficiente de chuva e precipitação mensal.

\section{MATERIAL E MÉTODOS}

Dados pluviográficos de 889 chuvas individuais erosivas ocorridas nos períodos de 1972 a 1975, 1977 a 1985 e 1995 a 1999 foram analizados, totalizando 18 anos de dados pluviográficos, no município de Cuiabá, MT, obtidos junto ao $9^{\circ}$ Distrito de Meteorologia do Instituto Nacional de Meteorologia (INMET). A estação meteorológica do município de Cuiabá está localizada na latitude $15^{\circ} 37^{\prime} 18^{\prime \prime} \mathrm{W}$ e longitude $56^{\circ} 06^{\prime} 30^{\prime \prime} \mathrm{W}$, numa altitude de $151,34 \mathrm{~m}$; a precipitação média é de 1.387,7 mm e o tipo climático predominante na região é o Aw de Koppen, isto é, Tropical semiúmido, com 4 a 5 meses secos e duas estações bem definidas, uma seca (outonoinverno) e uma chuvosa (primavera-verão), com características de continentalidade.

Obtiveram-se as chuvas individuais erosivas estudadas em pluviograma no qual são registrados a lâmina de chuva e o tempo de ocorrência; sua amplitude de registro varia de zero a $10,2 \mathrm{~mm}$ de precipitação, com unidade de $0,1 \mathrm{~mm}$, durante $24 \mathrm{~h}$, com sifonamento a cada $10 \mathrm{~mm}$.

Considerou-se chuva individual aquelas separadas da anterior e da posterior, por um período de no mínimo seis horas com chuvas de no máximo 1,0 $\mathrm{mm}$, e chuvas erosivas aquelas com quantidade igual ou superior a $10,0 \mathrm{~mm}$ ou igual ou superior a $6,0 \mathrm{~mm}$, desde que ocorridas em menos de 15 minutos, segundo os critérios propostos por Wischmeier \& Smith (1978), com modificações sugeridas por Carvalho et al. (1989).

A erosividade mensal, anual e média das chuvas, foi expressa pelo índice $\mathrm{EI}_{30}$ (Wischmeier \& Smith, 1958) no Sistema Internacional de Unidades - MJ mm ha ${ }^{-1} \mathrm{~h}^{-1}$ (Foster et al., 1981), obtida pelo produto entre a energia cinética total da chuva (E MJ ha $\left.{ }^{-1}\right)$ e a intensidade máxima em $30 \mathrm{~min}\left(\mathrm{I}_{30}-\mathrm{mm} \mathrm{h}^{-1}\right)$, de acordo com Wischmeier \& Smith (1958), e agrupadas por meses e anos. A energia cinética das chuvas foi determinada por meio da Eq. 1 Wischmeier \& Smith (1958), ajustada ao Sistema Internacional de Unidades por Foster et al. (1981).

$$
\mathrm{EC}=0,119+0,0873 \log \mathrm{Ip}
$$

em que: EC - é a energia cinética, $M J \mathrm{ha}^{-1} \mathrm{~mm}^{-1}$ e Ip - é a intensidade de precipitação da chuva, $\mathrm{mm} \mathrm{h}^{-1}$

Segundo Foster et al. (1981), o diâmetro das gotas de chuva não aumenta quando as intensidades são iguais ou superiores a $76 \mathrm{~mm} \mathrm{~h}^{-1}$; desta forma, para essas condições de intensidade de precipitação a energia cinética passa a ter valor máximo de $0,283 \mathrm{MJ} \mathrm{ha}^{-1} \mathrm{~mm}^{-1}$. A energia cinética acumulada de cada segmento de $10 \mathrm{~min}$ de uma chuva erosiva individual foi obtida pela aplicação da Eq. 2.

$$
\mathrm{EC}_{\mathrm{s}}=\mathrm{EC} \times \mathrm{h}
$$

em que: $\mathrm{EC}_{\mathrm{s}}$ - é a energia cinética no segmento de chuva, $\mathrm{MJ}$ $\mathrm{ha}^{-1} \mathrm{e} \mathrm{h}$ - é a quantidade de chuva no segmento uniforme, mm.

Obteve-se a energia cinética total da chuva erosiva individual pelo somatório da energia cinética de cada segmento 
da chuva, enquanto a intensidade máxima em trinta minutos foi obtida conforme a Eq. 3 proposta por Carvalho et al. (1989):

$$
\mathrm{I}_{30}=\frac{\left(\sum_{\mathrm{i}=1}^{\mathrm{n}} \mathrm{i}_{\mathrm{ni}} \times \mathrm{t}_{\mathrm{ni}}\right)}{30}
$$

em que: $\mathrm{I}_{30}$ - é a intensidade da chuva em 30 min consecutivos $\left(\mathrm{mm} \mathrm{h}^{-1}\right) ; \mathrm{i}_{\mathrm{n}}$ - é o valor da intensidade da chuva de ordem $\mathrm{n}(\mathrm{mm}$ $\mathrm{h}^{-1}$ ), ocorrida dentro do período de $30 \mathrm{~min} ; \mathrm{t}_{\mathrm{n}}$ - é o tempo de ocorrência da intensidade da chuva de ordem $n$, ocorrida dentro do período de $30 \mathrm{~min}$. Eq. 4:

O índice de erosividade $\left(\mathrm{EI}_{30}\right)$ de cada chuva foi obtido pela

$$
\mathrm{EI}_{30}=\mathrm{EC}_{\mathrm{t}} \times \mathrm{I}_{30}
$$

em que: $\mathrm{EI}_{30}$ - é o índice de erosividade da chuva erosiva individual, $\mathrm{MJ} \mathrm{mm} \mathrm{ha}^{-1} \mathrm{~h}^{-1} \mathrm{e} \mathrm{I}_{30}$ - é a intensidade máxima da chuva no período de $30 \mathrm{~min}$ consecutivos, $\mathrm{mm} \mathrm{h}^{-1}$.

Por sua vez, o $\mathrm{EI}_{30}$ mensal foi obtido pelo somatório do $\mathrm{EI}_{30}$ de cada chuva erosiva ocorrida no mês e o $\mathrm{EI}_{30}$ anual pelo somatório do $\mathrm{EI}_{30}$ de cada mês, cujo fator R foi representado pela média dos valores de $\mathrm{EI}_{30}$ anuais.

Nas análises de probabilidade de ocorrência e do período de retorno do índice $\mathrm{EI}_{30}$ determinados para os anos em estudo, foram utilizadas a lei de probabilidade logarítmica e a teoria de valores extremos preconizadas por Schwab et al. (1981). O período de retorno e a probabilidade de ocorrência foram obtidos pelas Eqs. 5 e 6, respectivamente.

$$
\begin{gathered}
\mathrm{TR}=\frac{(\mathrm{N}+1)}{\mathrm{m}} \\
\mathrm{Pr}=\frac{100}{\mathrm{TR}}
\end{gathered}
$$

em que: TR - é o período de retorno no qual o índice de erosividade é igualado ou superado; $\mathrm{N}$ - é o número de anos de registro de dados (tamanho da série); $\mathrm{m}$ - é o número de ordem do índice de erosividade quando a série de dados de erosividade é colocada em ordem decrescente de magnitude e $\mathrm{Pr}$ - é a probabilidade de ocorrência; em seguida, ajustou-se o modelo de distribuição de probabilidades de Gumbel para o cálculo anual das erosividades máximas por meio das Eqs. 7, 8 e 9, conforme Pinto et al. (1996):

$$
\begin{aligned}
& \mathrm{Y}_{\mathrm{TR}}=-\ln \left[-\ln \left(1-\frac{1}{\mathrm{TR}}\right)\right]_{\mathrm{t}} \\
& \mathrm{K}_{\mathrm{TR}}=-0,45+0,78 \times \mathrm{Y}_{\mathrm{TR}}
\end{aligned}
$$

$$
\mathrm{X}_{\mathrm{TR}}=\overline{\mathrm{X}}+\mathrm{S} \times \mathrm{K}_{\mathrm{TR}}
$$

em que: $\mathrm{Y}_{\mathrm{TR}}$ - é a variável reduzida da distribuição de Gumbel; $\mathrm{TR}$ - é o período de retorno (anos); $\mathrm{X}_{\mathrm{TR}}$ - é a erosividade máxima (anual ou mensal) para determinado TR $\left(\mathrm{MJ} \mathrm{mm} \mathrm{ha}^{-1} \mathrm{~h}^{-1} \mathrm{ano}^{-1}\right)$; $\mathrm{K}_{\mathrm{TR}}$ - é o fator de frequência (adimensional); $\mathrm{X}$ - é a média da erosividade máxima anual ou mensal $\left(\mathrm{MJ} \mathrm{mm} \mathrm{ha}{ }^{-1} \mathrm{~h}^{-1} \mathrm{ano}^{-1}\right)$ e $\mathrm{S}$ - é o desvio padrão dos dados de erosividade máxima (MJ mm $\left.\mathrm{ha}^{-1} \mathrm{~h}^{-1} \mathrm{ano}^{-1}\right)$.

A Eq. 8 é chamada Modelo Geral de Ven Te Chow. Essas equações permitem o emprego de uma população de dados e não apenas de uma amostra simplificando com isto as equações da distribuição de Gumbel.

Utilizou-se o teste de Kolmogorov-Smirnov com o objetivo de verificar a adequabilidade à distribuição de Gumbel para a representação probabilística dos dados.

Foi utilizado papel de probabilidade logarítmica para plotar os valores dos índices de erosividade observados, em que os valores dos índices anuais de erosividade, para qualquer período de retorno desejado, ou vice-versa, podem ser facilmente obtidos pela leitura direta na curva de distribuição de probabilidade ou calculados pela aplicação da equação dos valores teóricos. Para determinar o número adequado de anos de registro de chuvas visando ao cálculo da erosividade, utilizou-se a eq. 10, proposta por Mokus, relatada em Schwab et al. (1981):

$$
\mathrm{Y}=\left(4,30 \times \mathrm{t}_{\alpha} \times \log \theta\right)+6
$$

em que: Y - é o número mínimo de anos de chuva para o cálculo da erosividade; $\mathrm{t}$ - é o valor tabelado (distribuição "t" de Student); $\alpha$ - é o nível de significância, normalmente com $\mathrm{p}<$ 0,1 e com graus de liberdade Y-6 e $\theta$ - é a relação das magnitudes entre o evento com período de retorno de 100 anos e o evento com período de retorno de dois anos.

Utilizando-se dados pluviométricos do período de 1972 a 1975, 1977 a 1985 e 1995 a 1999, para cada mês do ano, calculouse o coeficiente de chuva, por meio do método proposto por Lombardi Neto (1977), cuja expressão é dada por:

$$
\mathrm{R}_{\mathrm{c}}=\frac{\mathrm{Pm}^{2}}{\mathrm{~Pa}}
$$

em que: Rc - é o coeficiente de chuva (mm); Pm - é a precipitação média mensal (mm) e Pa - é a precipitação média anual (mm).

Através do programa estatístico SPSS 15.0, procedeu-se ao ajuste dos dados de índices de erosividade médios mensais e de coeficiente de chuva às funções dos seguintes tipos: linear, potencial, exponencial, quadrática e logarítmica; desta forma, foram apresentados os coeficientes de ajuste para o modelo matemático que apresentou, na análise de variância, significância para regressão, para os parâmetros do modelo e maior coeficiente de determinação $\left(\mathrm{R}^{2}\right)$. 


\section{RESULTADOS E DISCUSSÃO}

$\mathrm{Na}$ Tabela 1 se apresentam os valores mensais, anuais e médios do índice de erosividade da chuva com base na série de dados pluviográficos do município de Cuiabá, MT. Os resultados mensais apresentaram coeficientes de variação elevados para os meses de maio, junho, julho e agosto. Tratando-se de meses de baixa precipitação na região de Cuiabá, referidos resultados podem ser considerados normais já que é comum uma variabilidade maior na ocorrência de chuvas.

$\mathrm{O}$ fator $\mathrm{R}$ de Cuiabá, determinado através do índice de erosividade $\mathrm{EI}_{30}$, foi de $8.810 \mathrm{MJ} \mathrm{mm} \mathrm{ha}^{-1} \mathrm{~h}^{-1} \mathrm{ano}^{-1}$, com medidas de dispersão de 2.244 e $529 \mathrm{MJ} \mathrm{mm} \mathrm{ha}^{-1} \mathrm{~h}^{-1}$ ano $^{-1}$, respectivamente, para o desvio-padrão e erro-padrão da média e com coeficiente de variação de $25 \%$ (Tabela 2); assim, o fator erosividade da chuva de Cuiabá ( $8.810 \mathrm{MJ} \mathrm{mm} \mathrm{ha}^{-1} \mathrm{~h}^{-1} \mathrm{ano}^{-1}$ ) foi superior aos de Teodoro Sampaio, SP, Piraju, SP, Campinas, SP, e Piracicaba, SP, obtidos, respectivamente, por Colodro et al. (2002), Lombardi Neto (1977), Pereira (1983), Sosa (1987) e Roque et al. (2001); no entanto, próximo ao fator R de Uruguaiana, RS, obtido por Hickmann et al. (2008) e inferior aos de São Borja, RS (Cassol et al., 2008) e de Santa Rosa, RS (Mazurana et al., 2009).

Os valores extremos anuais variaram de 3.820,72 a 13.712,20 $\mathrm{MJ} \mathrm{mm} \mathrm{ha}{ }^{-1} \mathrm{~h}^{-1} \mathrm{ano}^{-1}$, respectivamente, em 1985 e 1998, assim como os valores extremos do índice de erosividade médio mensal foram de 0 (meses de junho, julho e agosto) a $4.372 \mathrm{MJ}$ $\mathrm{mm} \mathrm{ha}^{-1} \mathrm{~h}^{-1}$ (janeiro de 1999).

A Figura 1 apresenta a distribuição dos valores médios mensais do índice de erosividade $\mathrm{EI}_{30}$ e da precipitação pluviométrica do município de Cuiabá, nos períodos de 1972 a
1975, 1977 a 1985 e 1995 a 1999. Os dados evidenciam que $86 \%$ da precipitação anual e $87 \%$ do índice de erosividade ocorrem de outubro a abril. Em termos relativos quanto absolutos, a erosividade da chuva para Cuiabá foi, nessa época, superior aos valores obtidos para o Estado de São Paulo (Carvalho et al., 1989; Colodro et al., 2002; Lombardi Neto, 1977; Sosa, 1987; Pereira, 1983; Roque et al., 2001) e do Rio de Janeiro (Gonçalves et al., 2006).

Na Figura 2 está apresentado o percentual acumulado do índice de erosividade e precipitação; esta curva de distribuição da erosividade da chuva fornece informações de grande importância para determinação do fator cobertura e manejo (C) da equação universal de perdas do solo e indica a época do ano em que ocorre o maior potencial das chuvas em causar erosão. Pode-se constatar que os meses de maior erosividade (outubro a abril) coincidem com o segmento de maior inclinação da curva, visto que, quanto maior a inclinação dessa curva para determinado período, maior também é o risco de erosão do solo necessitando-se, portanto, de maiores cuidados de proteção naquele período.

Observa-se uma harmonia entre as distribuições médias mensais do índice de erosividade e do volume de chuva precipitado no município de Cuiabá, MT. Nota-se, também, que alguns meses de alta erosividade coincidem com a época de preparo do solo e semeadura na região de Cuiabá. O alto potencial erosivo das chuvas nessas épocas do ano, aliado ao fato do solo se encontrar sem cobertura e desagregado, particularmente quando o preparo convencional (arações e gradagens) é utilizado, pode contribuir significativamente para grandes perdas de solo e água. Neste período o manejo do solo e das culturas deve procurar manter a cobertura vegetal, sendo um recurso o plantio direto, evitando a pulverização do

Tabela 1. Distribuição mensal e anual do índice de erosividade $\mathrm{El}_{30}$ para o município de Cuiabá, MT, nos períodos de 1972 a 1975,1977 a 1985 e 1995 a 1999

\begin{tabular}{|c|c|c|c|c|c|c|c|c|c|c|c|c|c|}
\hline \multirow{2}{*}{ Ano } & \multicolumn{13}{|c|}{ Índice de erosividade $\mathrm{El}_{30}\left[\mathrm{MJ} \mathrm{mm} \mathrm{ha}^{-1} \mathrm{~h}^{-1}\right]$} \\
\hline & Jan & Fev & Mar & Abr & Mai & Jun & Jul & Ago & Set & Out & Nov & Dez & Anual \\
\hline 1972 & 1481 & 948 & 345 & 1191 & 284 & 11 & 26 & 519 & 10 & 1147 & 1235 & 1351 & 8550 \\
\hline 1973 & 3682 & 70 & 380 & 63 & 0 & 0 & 0 & 127 & 149 & 289 & 349 & 1027 & 6135 \\
\hline 1974 & 2068 & 1197 & 1614 & 647 & 29 & 0 & 0 & 5 & 193 & 1147 & 1576 & 1351 & 9827 \\
\hline 1975 & 700 & 1078 & 524 & 305 & 0 & 6 & 0 & 361 & 368 & 2839 & 1576 & 1533 & 9290 \\
\hline 1977 & 1164 & 918 & 877 & 997 & 357 & 613 & 0 & 0 & 54 & 555 & 1576 & 4000 & 11111 \\
\hline 1978 & 1905 & 1591 & 2154 & 993 & 613 & 0 & 220 & 0 & 163 & 157 & 466 & 740 & 9003 \\
\hline 1979 & 707 & 542 & 2496 & 1490 & 437 & 0 & 10 & 3 & 157 & 270 & 654 & 276 & 7042 \\
\hline 1980 & 1298 & 2870 & 564 & 441 & 34 & 1 & 0 & 0 & 877 & 416 & 1549 & 1390 & 9439 \\
\hline 1981 & 803 & 799 & 1299 & 148 & 20 & 199 & 0 & 0 & 137 & 764 & 709 & 2858 & 7736 \\
\hline 1982 & 2328 & 831 & 902 & 1384 & 576 & 0 & 210 & 55 & 454 & 206 & 980 & 1086 & 9013 \\
\hline 1983 & 1407 & 529 & 1020 & 570 & 251 & 0 & 16 & 0 & 387 & 516 & 3149 & 847 & 8691 \\
\hline 1984 & 224 & 1409 & 1076 & 839 & 304 & 0 & 0 & 16 & 166 & 911 & 744 & 1323 & 7011 \\
\hline 1985 & 474 & 1017 & 147 & 355 & 54 & 0 & 0 & 425 & 94 & 666 & 208 & 381 & 3821 \\
\hline 1995 & 625 & 67 & 414 & 112 & 41 & 244 & 0 & 215 & 595 & 1210 & 757 & 2285 & 6565 \\
\hline 1996 & 2558 & 2943 & 5 & 24 & 1 & 4 & 0 & 982 & 794 & 1325 & 749 & 1871 & 11257 \\
\hline 1997 & 1385 & 256 & 208 & 316 & 677 & 1223 & 0 & 0 & 1197 & 1750 & 1810 & 1491 & 10313 \\
\hline 1998 & 1701 & 578 & 75 & 5 & 1597 & 0 & 0 & 1812 & 281 & 2291 & 3472 & 1901 & 13712 \\
\hline 1999 & 4372 & 1598 & 0 & 646 & 16 & 1002 & 0 & 0 & 1375 & 763 & 173 & 129 & 10072 \\
\hline $\mathrm{Md}^{1}$ & 1605 & 1069 & 783 & 585 & 294 & 183 & 27 & 251 & 414 & 957 & 1207 & 1436 & 8810 \\
\hline $\mathrm{DP}^{2}$ & 1099 & 807 & 727 & 473 & 400 & 373 & 69 & 470 & 401 & 734 & 920 & 941 & 2244 \\
\hline $\mathrm{EPM}^{3}$ & 259 & 190 & 171 & 111 & 94 & 88 & 16 & 111 & 95 & 173 & 217 & 222 & 529 \\
\hline C.V. ${ }^{4}$ & 68 & 76 & 93 & 81 & 136 & 203 & 257 & 187 & 97 & 77 & 76 & 66 & 25 \\
\hline
\end{tabular}


Tabela 2. Probabilidade de ocorrência e período de retorno dos valores dos índices anuais de erosividade $\left(\mathrm{El}_{30 \text { anual }} \mathrm{M}\right)$ $\mathrm{mm} \mathrm{ha-1} \mathrm{h}^{-1} \mathrm{ano}^{-1}$ ) e mês de ocorrência da chuva mais erosiva do ano no município de Cuiabá, MT no período de 1972 a 1975, 1977 a 1985 e 1995 a 1999

\begin{tabular}{|c|c|c|c|c|c|c|}
\hline \multirow[t]{2}{*}{ Ano } & \multirow{2}{*}{$\begin{array}{c}\text { El }_{\text {30anual }} \\
\text { MJ } \mathrm{mm} \mathrm{ha}^{-1} \mathrm{~h}^{-1}\end{array}$} & \multirow[t]{2}{*}{$\mathrm{m}^{1}$} & \multirow[t]{2}{*}{$\mathrm{T}^{2}$} & \multirow[t]{2}{*}{$\mathbf{P}^{3}$} & \multicolumn{2}{|c|}{$\begin{array}{l}\text { Mês de ocorrência da } \\
\text { chuva mais erosiva do } \\
\text { ano }\end{array}$} \\
\hline & & & & & Mês & $\begin{array}{c}E_{30 \text { mensal }} \\
\text { MJ mm ha }{ }^{-1} h^{-1}\end{array}$ \\
\hline 1998 & 13.712 & 1 & 19,00 & 5,26 & Nov. & 3.472 \\
\hline 1996 & 11.257 & 2 & 9,50 & 10,53 & Fev. & 2.943 \\
\hline 1977 & 11.111 & 3 & 6,33 & 15,79 & Dez. & 4.000 \\
\hline 1997 & 10.313 & 4 & 4,75 & 21,05 & Nov. & 1.810 \\
\hline 1999 & 10.072 & 5 & 3,80 & 26,32 & Jan. & 4.372 \\
\hline 1974 & 9.827 & 6 & 3,17 & 31,58 & Jan. & 2.068 \\
\hline 1980 & 9.439 & 7 & 2,71 & 36,84 & Fev. & 2.870 \\
\hline 1975 & 9.290 & 8 & 2,38 & 42,11 & Out. & 2.839 \\
\hline 1982 & 9.013 & 9 & 2,11 & 47,37 & Jan. & 2.328 \\
\hline 1978 & 9.003 & 10 & 1,90 & 52,63 & Mar. & 2.154 \\
\hline 1983 & 8.691 & 11 & 1,73 & 57,89 & Nov. & 3.149 \\
\hline 1972 & 8.550 & 12 & 1,58 & 63,16 & Jan. & 1.481 \\
\hline 1981 & 7.736 & 13 & 1,46 & 68,42 & Dez. & 2.858 \\
\hline 1979 & 7.042 & 14 & 1,36 & 73,68 & Mar. & 2.496 \\
\hline 1984 & 7.011 & 15 & 1,27 & 78,95 & Fev. & 1.409 \\
\hline 1995 & 6.565 & 16 & 1,19 & 84,21 & Dez. & 2.285 \\
\hline 1973 & 6.135 & 17 & 1,12 & 89,47 & Jan. & 3.682 \\
\hline 1985 & 3.821 & 18 & 1,06 & 94,74 & Fev. & 1017 \\
\hline$M d^{4}$ & 8.810 & & & & & \\
\hline$D P^{5}$ & 2.244 & & & & & \\
\hline $\mathrm{Epm}^{6}$ & 529 & & & & & \\
\hline $\mathrm{CV}^{7}$ & 25 & & & & & \\
\hline
\end{tabular}

desvio padrão; 6 - erro padrão médio; 7 - coeficiente de variação, \%

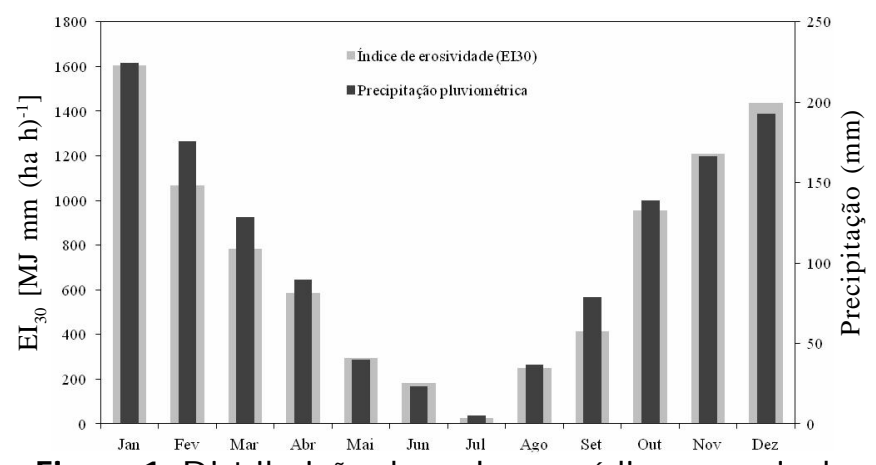

Figura 1. Distribuição dos valores médios mensais do índice de erosividade $\left(\mathrm{El}_{30}\right)$ e da precipitação pluviométrica do município de Cuiabá, MT, nos períodos de 1972 a 1975, 1977 a 1985 e 1995 a 1999

solo, a formação de crosta de permeabilidade reduzida e a compactação subsuperficial, procurando variar o tipo e a profundidade de utilização dos implementos ao longo do tempo.

$\mathrm{Na}$ Tabela 2 se encontram o período de retorno, a probabilidade de ocorrência e o mês mais crítico dos valores dos índices de erosividade observados para o município de Cuiabá. Os valores do período de retorno e da probabilidade de ocorrência determinados para o maior índice de erosividade anual observado $\left(13.712,20 \mathrm{MJ} \mathrm{mm} \mathrm{ha}^{-1} \mathrm{~h}^{-1}\right.$ ano $\left.^{-1}\right)$ foram de 19 anos e 5,26\%, respectivamente. Portanto, prevê-se neste local, com probabilidade de 5,26\%, um valor do índice anual de

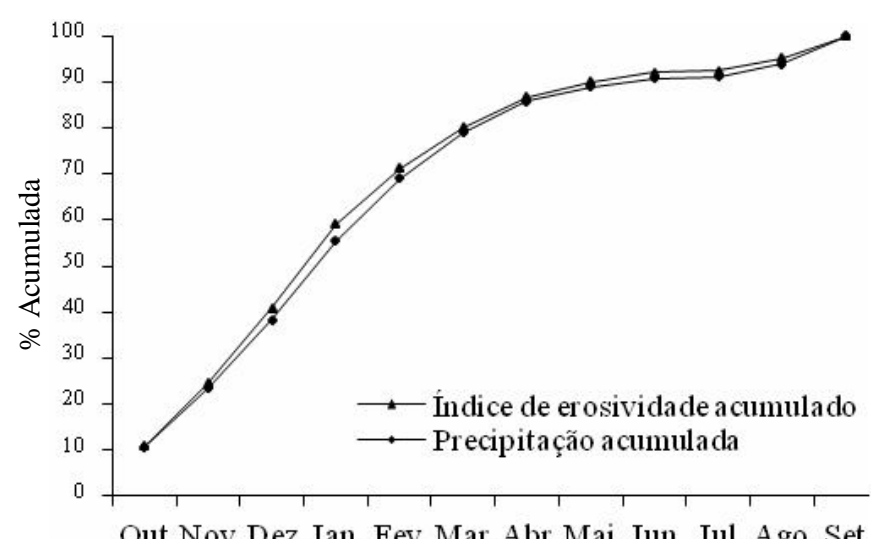

Out Nov Dez Jan Fev Mar Abr Mai Jun Jul Ago Set Figura 2. Distribuição relativa acumulada do índice de erosividade $\left(\mathrm{El}_{30}\right)$ médio mensal e precipitação do município de Cuiabá, MT, nos períodos de 1972 a 1975, 1977 a 1985 e 1995 a 1999

erosividade igual ou superior a $13.712,20 \mathrm{MJ} \mathrm{mm} \mathrm{ha}^{-1} \mathrm{~h}^{-1}$ ano $^{-1}$ pelo menos uma vez a cada 19 anos. Para o menor índice de erosividade anual observado $\left(3.820,72 \mathrm{MJ} \mathrm{mm} \mathrm{ha}^{-1} \mathrm{~h}^{-1}\right.$ ano $\left.^{-1}\right)$, os valores foram, respectivamente, de 1,06 ano e de $94,74 \%$, razão pela qual se espera, com probabilidade de $94,74 \%$, um valor do índice anual de erosividade igual ou superior a 3.820,72 MJ mm ha ${ }^{-1} \mathrm{~h}^{-1}$ ano $^{-1}$, pelo menos uma vez a cada 1,06 ano.

Com relação aos valores da erosividade mensal obteve-se, para o mês de janeiro de 1999, o valor máximo de $\mathrm{EI}_{30}(4.372 \mathrm{MJ}$ $\mathrm{mm} \mathrm{ha}^{-1} \mathrm{~h}^{-1}$ ) para o período de tempo estudado, esperando-se que o mesmo possa ser igualado ou superado, pelo menos uma vez a cada 19 anos, com probabilidade de ocorrência de $5,26 \%$.

A curva de distribuição de probabilidade dos valores anuais (Figura 3A) e máximos mensais (Figura 3B) de erosividade para Cuiabá (MT) seguiu o padrão Gumbel, simplificada por Ven Te Chow, típico de eventos extremos de precipitação (Pinto, 1996). Os valores dos índices anuais e mensais de erosividade para qualquer outro período de retorno desejado, ou vice-versa, podem ser facilmente obtidos através da leitura direta na curva de distribuição de probabilidade apresentadas nas Figuras 3 e 4, respectivamente, ou calculados através da aplicação da Eq. 8; observa-se, assim, que o período de retorno do fator erosividade da chuva $(\mathrm{R})$ do município de Cuiabá (8.810 MJ $\mathrm{mm} \mathrm{ha}^{-1} \mathrm{~h}^{-1}$ ano $^{-1}$ ) foi de 2,33 anos, equivalente a uma probabilidade de ocorrência de $42,92 \%$, ou seja, o valor erosividade média de Cuiabá ocorre pelo menos uma vez a cada 2,33 anos com probabilidade de $42,92 \%$.

Este período de retorno encontrado para o fator R para o município de Cuiabá foi semelhante àqueles obtidos para os fatores R de Campinas (2,1 anos), Piracicaba (2,1 anos), Pindorama (2,1 anos), Mococa (2,4 anos), Botucatu (2,3 anos), Selvíria (2,3 anos), Teodoro Sampaio (2,3 anos) e Piraju (2,3) determinados por Lombardi Neto (1977), Pereira (1983), Sosa (1987), Carvalho et al. (1989; 2004), Moreti et al. (2003a), Colodro et al. (2002) e Roque et al. (2001), respectivamente.

Cabe ressaltar que, embora se observe semelhança entre o período de retorno e a probabilidade de ocorrência do fator $\mathrm{R}$ no presente trabalho com as localidades supracitadas, o valor do fator $\mathrm{R}$ obtido neste trabalho se distingue daqueles 

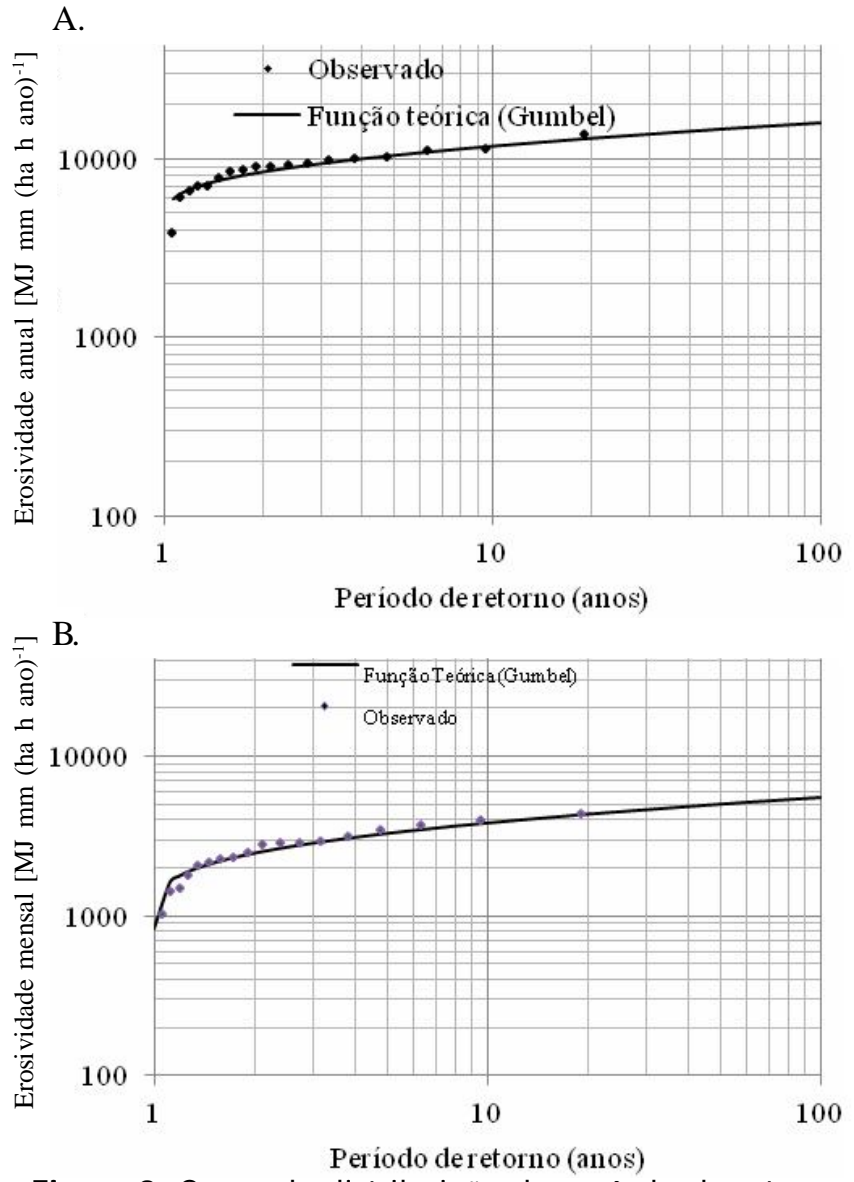

Figura 3. Curva de distribuição do período de retorno dos índices anuais (A) e mensais (B) de erosividade da chuva do município de Cuiabá, MT, nos períodos de 1972 a 1975, 1977 a 1985 e 1995 a 1999

encontrados nos trabalhos referidos anteriormente, evidenciando, assim, a grande importância de se realizar este tipo de caracterização para cada localidade e/ou região, sendo muito perigoso usar valores de outras regiões ou localidades uma vez que essas localidades do Estado de São Paulo já listadas e embora possuam tipo climático diferente da cidade de Cuiabá, têm regime pluviométrico bem próximo e, mesmo assim, possuem valores de erosividade, período de retorno e probabilidade de ocorrências distintos.

Os valores dos índices de erosividade anual para os períodos de retorno de 2, 5, 10, 20, 50 e 100 anos, foram de 8.442, 10.426, $11.739,12.999,14.630,15.852 \mathrm{MJ} \mathrm{mm} \mathrm{ha}^{-1} \mathrm{~h}^{-1}$ ano $^{-1}$. Os valores máximos anuais do índice de erosividade mensal, esperados para os respectivos períodos de retorno foram, respectivamente, de 2.475, 3.280, 3.813, 4.324, 4.986 e 5.482 $\mathrm{MJ} \mathrm{mm} \mathrm{ha}^{-1} \mathrm{~h}^{-1} \mathrm{ano}^{-1}$, segundo o método preconizado em Pinto (1996), ou seja, todos os valores esperados tanto anuais como mensais, foram obtidos a partir da aplicação da função de Gumbel ajustada.

$\mathrm{A}$ adequação do número de anos de registro de dados de erosividade é de 10 e 16 anos como tempo mínimo aceitável de dados para obtenção do fator R local, com nível de significância de 10 e $2 \%$, respectivamente, de acordo com a indicação de Schwab et al. (1981).

As análises mostraram, para Cuiabá, alta correlação do índice de erosividade $\mathrm{EI}_{30}$ com a precipitação $(\mathrm{r}=0,833)$ e com o coeficiente de chuva $(r=0,811)$. O modelo de regressão potencial entre o índice de erosividade $\mathrm{EI}_{30}$ médio mensal e os parâmetros de pluviometria (precipitação - Figura 4A e coeficiente da chuva - Figura 4B) apresentou melhor ajuste, com coeficiente de determinação elevado, altamente significativos $(\mathrm{p}<0,0001)$.
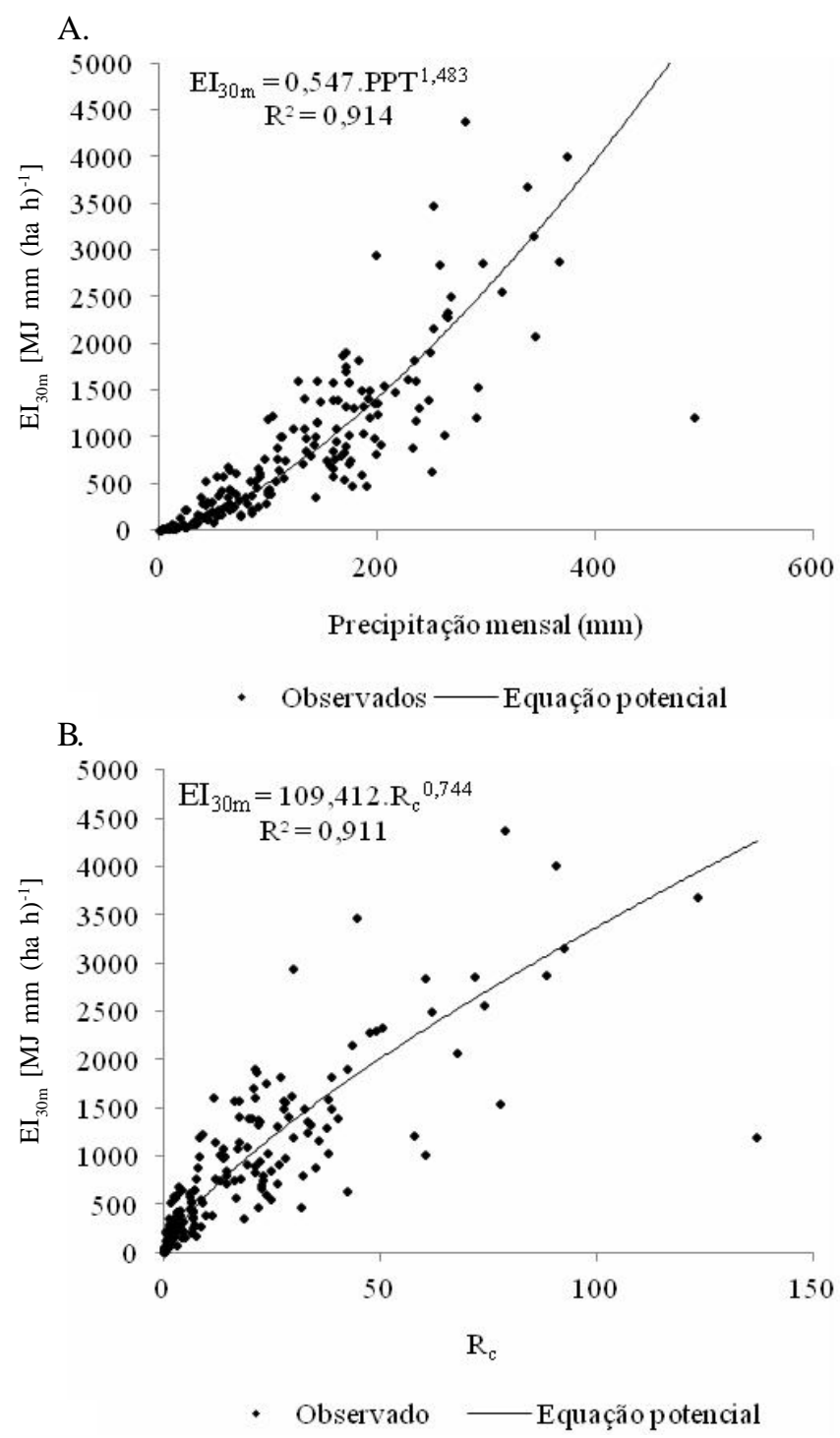

Figura 4. Regressão potencial entre o índice de erosi vidade médio mensal $\left(\mathrm{El}_{30 \mathrm{~m}}\right)$ e a precipitação média mensal $\mathrm{PPT}_{m}(\mathrm{~A})$ e o coeficiente de chuva médio mensal - Rc (B) no município de Cuiabá/M T nos períodos de 1972 a 1975, 1977 a 1985 e 1995 a 1999

O melhor ajuste do modelo potencial para se fazer a estimativa do índice de erosividade da chuva a partir de dados pluviométricos, também foi observado para outras localidades como, por exemplo, Campinas, Piracicaba, Pindorama Piraju e Mococa, em São Paulo por Lombardi Neto (1977), Pereira (1983), Sosa (1987), Roque et al. (2001) e Carvalho (1989), embora tenha contratado com os dados de Lages e de Campos Novos, SC e Teodoro Sampaio, SP, observados, respectivamente, por Bertol (1993; 1994) e Colodro et al. (2002), cujo modelo de melhor ajuste foi linear. 


\section{CONCLUSÕES}

1. O fator R da Equação Universal da Perda de Solo para o município de Cuiabá, MT, é de $8.810 \mathrm{MJ} \mathrm{mm} \mathrm{ha}^{-1} \mathrm{~h}^{-1} \mathrm{ano}^{-1}$, sendo classificado como erosividade alta. Este fator possui probabilidade de ocorrência de $42,92 \%$, podendo ser igualado ou superado pelo menos uma vez a cada 2,33 anos.

2. Cerca de $87 \%$ do total da erosividade anual se distribuem nos meses de outubro a abril indicando que, neste período, é prevista a maior parte das perdas anuais de solo por erosão.

3. O modelo matemático potencial apresenta bom ajuste para descrever a relação entre o $\mathrm{EI}_{30}$ mensal e o coeficiente de chuva $\left(R^{2}=0,91\right)$ e a precipitação mensal $\left(R^{2}=0,91\right)$.

\section{LITERATURA CITADA}

Bertol, I. Índice de erosividade $\left(\mathrm{EI}_{30}\right)$ para Lages $(\mathrm{SC})-1^{\mathrm{a}}$. aproximação. Pesquisa Agropecuária Brasileira, v.28, p.515$521,1993$.

Bertol, I. Avaliação da erosividade da chuva na localidade de Campos Novos (SC) No período de 1981-1990. Pesquisa Agropecuária Brasileira, v.29, p.1453-1458, 1994.

Carvalho, D. F. de ; Machado, R. L. ; Evangelista, A. W. P. ; Khoury Júnior, J. K. ; Silva, L. D. B. da. Distribuição, probabilidade de ocorrência e período de retorno dos índices de erosividade EI30 e KE> 25 em Seropédica - RJ. Engenharia Agrícola v.30, p.245-252, 2010.

Carvalho, M. P.; Lombardi Neto, F.; Vasques Filho, J.; Cataneo, A. İndices de erosividade da chuva correlacionada com as perdas de um Podzólico Vermelho-amarelo eutrófico textura argilosa/muito argilosa de Mococa (SP): Primeira aproximação do fator erodibilidade dos solos. Revista Brasileira de Ciência do Solo, v.13 p.237-242, 1989.

Carvalho, M. P. E ; Freddi, O. S.; Veronese Junior, V. Critérios para classificação de chuva individual erosiva para o estado de São Paulo. Acta Scientiarum, v.26, p.175-183, 2004.

Cassol, E. A.; Eltz, F. L. F.; Martins, D.; Lemos, A. M.; Lima, V. S.; Bueno, A. C. Erosividade, padrões hidrológicos, período de retorno e probabilidade de ocorrência das chuvas de São Borja, RS. Revista Brasileira de Ciência do Solo, v.32, p.1239-1251, 2008.

Colodro, G.; Carvalho, M. P.; Roque, C. G.; Prado, R. M. Erosividade da chuva: distribuição e correlação com a precipitação pluviométrica de Teodoro Sampaio (SP). Revista Brasileira de Ciência do Solo, v.26, p.809-818, 2002.

Foster, G. R.; Mccool, D. K.; Renard, K. G.; Moldenhauer, W. C. Conversion of the universal soil loss equation to SI units. Journal of Soil and Water Conservation, Baltimore, v.36, p.355-359, 1981.

Gonçalves, F. A. ; Silva, D. D. ; Pruski, F. F. ; Carvalho, D. F. ; Cruz, E. S. Índices e espacialização da erosividade das chuvas para o Estado do Rio de Janeiro. Revista Brasileira de Engenharia Agrícola e Ambiental, v.10, p.269-276, 2006.
Hernani, L. C.; Freitas, P. L.; Pruski, F. F.; De Maria, I. C.; Castro Filho, C.; Landers, J. C. A erosão e seu impacto. In: Manzatto, C. V.; Freita Júnior, E.; Peres, J. R. R. Uso agrícola dos solos brasileiros. Rio de Janeiro; Embrapa Solos, 2002. p.47-60.

Hickmann, C.; Eltz, F. L. F.; Cassol, E. A.; Cogo, C. M. Erosividade das chuvas em Uruguaiana, RS, determinadas pelo índice $\mathrm{EI}_{30}$, com base no período de 1963 a 1991. Revista Brasileira de Ciência do Solo, v.32,.825-831, 2008.

Lombardi Neto, F. Rainfall erosivity - its distribution and relationship with soil loss as Campinas, Brasil. West Lafayette: Purdue University, 1977. 53p. Dissertação Mestrado

Mazurana, J.; Cassol, E. A.; Santos, L. C. dos; Eltz, F. L. F.; Bueno, A. C. Erosividade, padrões hidrológicos e período de retorno das chuvas erosivas de Santa Rosa (RS). Revista Brasileira de Engenharia Agrícola e Ambiental, v.13, p.975983, 2009.

Morais, L. F. B.; Silva, V.; Naschenveng, T. M. C.; Hardoin, P. C.; Almeida, J. E. L.; Weber, O. L. S.; Boel, E.; Durigon, V. Índice $\mathrm{EI}_{30}$ e sua relação com o coeficiente de chuva do sudoeste do Mato Grosso. Revista Brasileira de Ciência do Solo, v.15, p.339-344, 1991.

Moreti, D.; Carvalho, M. P.; Mannigel, A. R.; Medeiros, L. R. Importantes características de chuva para a conservação do solo e da água no município de São Manuel - SP. Revista Brasileira de Ciência do Solo, v.27, n.4, p.713-725, 2003a.

Moreti, D.; Mannigel, A. R. ; Carvalho, M. de P. e . Fator erosividade da chuva para o município de Pedrinhas Paulista, Estado de São Paulo. Acta Scientiarum (UEM), v.25, p. 137145, 2003b.

Pereira, H. H. G. Índice de erosividade de chuvas: distribuição e relações com a precipitação em Piracicaba (SP). Piracicaba, ESALQ/USP: 1983. 70p. Dissertação Mestrado

Pinto, F. A., Ferreira, P. A., Pruski, F. F., Alves, A. R., Cecon, P. R. Estimativa de chuvas intensas no Estado de Minas Gerais utilizando registros diários. Engenharia Agrícola, v.16, p.821, 1996.

Roque, C. G.; Carvalho, M. P.; Prado, R. M. Fator erosividade da chuva de Piraju (SP): Distribuição, probabilidade de ocorrência, período de retorno e correlação com o coeficiente de chuva. Revista Brasileira de Ciência do Solo, v.25, p.147156, 2001.

Schwab, G. O.; Frevert, R. K.; Edminster, T. W.; Barnes, K. K. Soil and water conservation engineering. 3.ed. New York: John Wiley \& Sons, 1981. 525p.

Sosa, D. A. Erosividade da chuva. Distribuição e correlação com as perdas de solo para Pindorama, São Paulo, Brasil. Piracicaba: ESALQ/USP, 1987. 105p. Dissertação Mestrado

Wischmeier, W. H.; Smith, D. D. Rainfall energy and its relationship to soil loss. Transaction American Geophysical Union, v.39, p.285- 291, 1958.

Wischmeier, W. H.; Smith, D. D. Predicting rainfall erosion losses: a guide to conservation planning. Washington: USDA, 58p. 1978. Agriculture Handbook 537. 